\title{
Robotic bronchial sleeve resections: technical details and early results
}

\author{
Marion Durand \\ Thoracic Unit, Ramsay Générale de Santé, Hôpital Privé d'Antony, Antony 92160, France. \\ Correspondence to: Dr. Marion Durand, Thoracic Unit, Hôpital Privé d'Antony, 1 rue Velpeau, Antony 92160, France. \\ E-mail: durandm@me.com
}

How to cite this article: Durand M. Robotic bronchial sleeve resections: technical details and early result. Mini-invasive Surg 2019;3:35. http://dx.doi.org/10.20517/2574-1225.2019.31

Received: 30 Aug 2019 First Decision: 18 Nov 2019 Revised: 18 Nov 2019 Accepted: 20 Nov 2019 Published: 29 Nov 2019

Science Editor: Valérie Lacroix Copy Editor: Jing-Wen Zhang Production Editor: Tian Zhang

\begin{abstract}
Aim: We report our four-arm robotic bronchial sleeve anatomical lung resection technique and its early results.
\end{abstract}

Methods: We retrospectively collected all the four-arm robotic sleeve anatomical lung resections we performed in our institution from February 2014 to August 2019. We reported the results as a series of cases.

Results: During that period, 582 robotic procedures were performed by a single surgeon, of which 486 were major anatomical lung resections. From this group, 10 patients (2\%) underwent bronchial sleeve resections. All patients were treated on the right lung. Neither conversion nor major events occurred during surgery. The first bronchial sleeve was performed for Patient 219. The mean length of procedure was 164 ( \pm 43) min. One patient died during hospitalization due to a non-related complication (gastric massive bleeding). Three patients had no complications. Six had minor complications (Clavien Dindo Grade 2) resulting in prolonged length of stay. The mean length of stay was 10 ( \pm 5.7 ) days. No bronchial fistula occurred. All resection margins were RO.

Conclusion: Four-arm robotic bronchial sleeve is a feasible and safe procedure. Telemanipulation surgery offers excellent technical conditions to ensure a hand-sewed anastomosis and RO resection. The technical principle and dissection are the same as those of open surgery. Patient selection and mastering of the telemanipulation device are mandatory to perform these complex and rare procedures.

Keywords: Lung carcinoma, robotic, surgery, sleeve lobectomy, sleeve segmentectomy

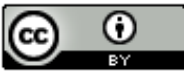

(C) The Author(s) 2019. Open Access This article is licensed under a Creative Commons Attribution 4.0 International License (https://creativecommons.org/licenses/by/4.0/), which permits unrestricted use, sharing, adaptation, distribution and reproduction in any medium or format, for any purpose, even commercially, as long as you give appropriate credit to the original author(s) and the source, provide a link to the Creative Commons license, and indicate if changes were made. 


\section{INTRODUCTION}

Telemanipulation surgery is a significant revolution in thoracic surgery. It allows minimizing the chest trauma while preserving-or enhancing-the surgeon's skills and vision, allows bimanual surgery, provides a $3 \mathrm{rd}$ hand, and gives $3 \mathrm{D}$ magnified vision. This is of greatest interest for complex procedures that are performed to spare the patient's lung function. Two trends are noticed in this area: the merger of sublobar resections ${ }^{[1]}$ and bronchial sleeve resections ${ }^{[2]}$. These 2 approaches require advanced skills that can be provided by the telemanipulator.

In this paper, we focus on the technical details of bronchial sleeve resections and report the early results of our experience.

\section{METHODS}

We collected retrospectively all the bronchial sleeve procedures performed in our center from the beginning of our robotic program in February 2014 to August 2019. All procedures were performed by a single surgeon. We analyzed them as a series of cases.

\section{Surgical technique}

The procedures were performed with either the Da Vinci $\mathrm{S}^{\mathrm{TM}}$ system or the $\mathrm{X}{ }^{\mathrm{TM}}$ system (Intuitive Surgical California). For the $\mathrm{S}^{\mathrm{TM}}$ system, a $12-\mathrm{mm} 30^{\circ}$ camera was used. For the $\mathrm{X}^{\mathrm{TM}}$ system, an $8-\mathrm{mm} 30^{\circ} \mathrm{camera}$ was used.

\section{Patient position and port placement}

The same patient position and port placement as for any robotic anatomical lung resection and node harvest were used, as described previously ${ }^{[3,4]}$. This is shown in Figure 1 and summarized below.

The patient was placed on their left side with a tissue roll below their chest to avoid the hip. The patient's body was stabilized with a vacuum cover. The right arm was placed in front of the head on the operating table. Neither central venous line nor arterial blood line was placed. A two-level paravertebral block and a serratus block were performed by the anesthesiologist with ultrasound guidance before surgical incision.

First, the design of the port placement was prepared. The shape of the scapula tip and scapula line were drawn. Then, the intercostal space (ICS) count was done from the 11th ICS from the back of the patient to the anterior side to spot the ninth for the $15-\mathrm{mm}$ port access and the 8 th for the camera port at the junction of the scapula line. The first port placed was the camera port to check the position of the other ports from inside the chest. After insertion of the camera, the capnothorax was started under vision control, and low pressure $(5 \mathrm{mmHg})$ and medium flow $(10 \mathrm{~L} / \mathrm{min})$ were applied. The other ports were placed in the following order: the right hand, the left hand, the third hand, and the port access.

The $30^{\circ}$ camera was inserted with vision up to place the other ports. The right-hand port was placed in the 7th ICS, at the junction of the diaphragm and the end of the major fissure. The left-hand port was placed in the 9th or 10th ICS above the triangular ligament. The 3rd hand was placed in the 7th ICS, at least 2 fingers closer to the spine to avoid conflict with the left hand, and at the junction of the visible muscular part of the ICS muscle and the posterior ICS ligament. Its angle of penetration in the chest was $90^{\circ}$. Then, the $15-\mathrm{mm}$ port access was placed in the ninth ICS at the diaphragm insertion, as low as possible to enlarge the triangle among it, the right hand, and the camera port. Then, the capnothorax insufflation was moved from camera port to port access.

\section{Instruments and procedure steps}

The instruments used for the procedures and for a right-handed surgeon were as follows:

- The right hand: permanent cautery spatula (Ref. 420184), needle holder SutureCut ${ }^{\mathrm{TM}}$ (Ref. 420296), or 


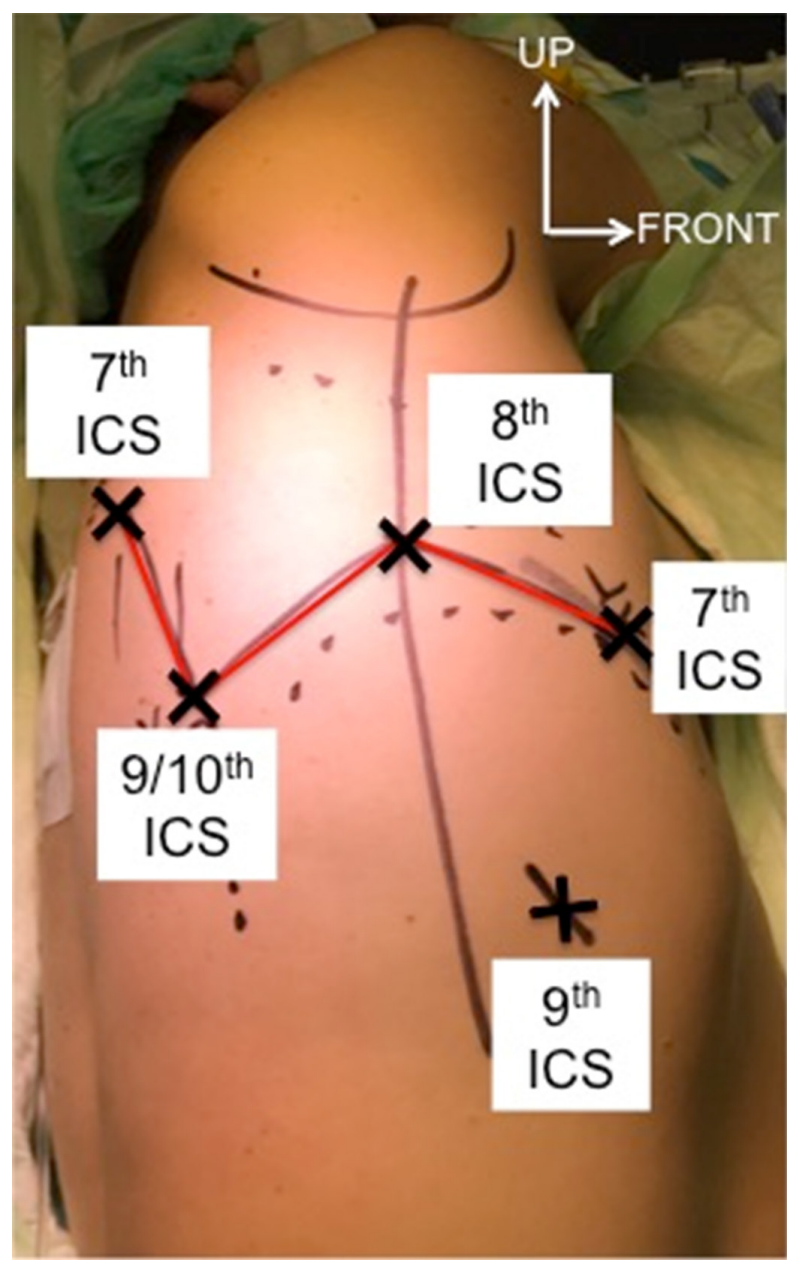

Figure 1. Port placement according to ICS. The red lines show the partial W design of the port placement; the interrupted lines show the projected major fissure (up) and diaphragm (down). ICS: inter costal space

curved scissors (Ref. 420178).

- The left hand: fenestrated bipolar forceps (Ref. 420205).

- The third hand (assistant arm): ProGrasp ${ }^{\mathrm{TM}}$ forceps (Ref. 420093).

Two handmade rolled gauzes were inserted, one for each grasper, to ensure a non-direct traction or lung exposure. A fissure-first technique and sharp dissection were performed. The steps were almost the same as routine lobectomy, mainly stated as follows:

- Triangular ligament opening and zone 8/9 node harvest.

- Spot of inferior pulmonary vein.

- Zone 7 node harvest and opening the posterior interbronchial zone.

- Spot the artery in the fissure.

- Open the fissure.

- Control and section of the artery (or arteries).

- Control and section of the vein.

- Finish the remaining parenchymal section.

- Bronchus dissection at last and section in pathologic zone.

- Placement of the lobe or segment in the bag.

- Complementary section of the bronchus in healthy macroscopic margin (and then sent to frozen section).

- Bronchial anastomosis. 
- Zone $2 \mathrm{R} 4 \mathrm{R}$ node harvest.

- Bag extraction.

- Chest tube placement and closing.

Arterial ligation was performed either by sewing 2 knots with linen 0 with the needle holder or by stapling with white $35 \mathrm{~mm}$ endo stappler [mainly for anterior mediastinal artery in case of right upper lobectomy (RUL)]. Venous ligation was performed either by sewing 1 knot with linen 0 doubled by a Vicryl 2/0 (22-mm needle) 10 -cm suture with the needle holder or by stapling with white $35 \mathrm{~mm}$ endo GIA. In the case of "manual" ligation of vessels, the distal part was dissected as far as possible and the section was done by spatula burning along the forceps and then distal vessel bipolar burn. Radical hilar and mediastinal node harvest were performed during the procedure.

The assistant was holding a long suction device (Elefant ${ }^{\oplus}$ Coloplast Ltd UK) to ensure a bloodless field and to avoid smoke inside the chest. The suction device was also used to stabilize the operating field by being placed over one of the rolled gauzes. For each procedure, a frozen section analysis of the bronchus border was performed to ensure the Ro margin. The specimen was placed after resection of the bronchus in an Endobag ${ }^{\circ}$ to prevent the chest contamination, and was extracted through the port access enlargement at the end of the procedure.

A 24 French chest drain was left in the chest through the right-hand port and minor suction was applied (minus $10 \mathrm{~cm}$ of water) after the patient's extubation.

\section{Sleeve lobectomy}

For the end-to-end anastomosis, V-Loc Covidien ${ }^{\mathrm{TM}}$ 3/0 180 (17-mm needle taper point, $15 \mathrm{~cm}$ length) sutures were used. For each anastomosis, 2 half-continuous sutures were performed.

The principle of the anastomosis technique, referring to a sleeve RUL, is as follows. A vertical axis exposure of the 2 borders was preferred. The posterior wall running suture started from outside the upper border, forehand, 3 o'clock, clockwise. After the first way out from the lower border, usually backhand, the needle was placed through the final loop of the wire to block the end of the running suture. The running suture was continued, mainly backhand, until 9 o'clock outside the lower border with the tension of the suture applied after each loop. Then, the anterior wall sewing was started with another V-Lock wire. The start of this second suture was from the lower border outside 3 o'clock, forehand. As with the previous first loop, the needle was placed through the final loop of the wire to block the end of the running suture after emerging from the upper border outside. The running suture was then conducted anti-clockwise to 9 o'clock outside the upper border, here again mainly backhand.

The airtightness was checked under water with mechanical insufflation before knotting the two wire ends, to ensure a harmonious tension of the running sutures. Then, the final knot was done and the needles were removed from the chest.

Regarding the lobe removal, exposure and gests were adapted. For a sleeve median lobectomy (ML), the bronchial section was done through a fissure exposure after pulling back the lower lobe artery with a loop (silicone 10-cm cut blue loop) to expose the intermediate bronchial trunk. The anastomosis was performed after changing the exposure for a posterior view. Then, the 2 borders were naturally placed to avoid a twist and the artery was hidden away from the sewing zone.

For inferior bilobectomy or lobectomy, the end anastomosis sleeve required a v-shaped cut of the distal part of the bronchus. Then, separate single knots were placed, using violet Vicryl 2/0 (22-mm needle, 10-cm 
length). Four to six wires were placed before starting to knot. A double loop was performed for the 1st knot to help tighten the knot.

For superior bilobectomy, pericardium section below the inferior pulmonary vein was achieved with the spatula to release the tension of the anastomosis. This could also be performed for RUL.

\section{S6 sleeve segmentectomy}

For sleeve segmentectomy, green light $\left(\right.$ FireFly $^{\mathrm{TM}}$ ) was used to ensure accurate parenchymal margin resection. After section of vascular and bronchial structure, $8 \mathrm{~mL}$ (i.e., $15 \mathrm{mg}$ of indocyanine green) were injected as an iv flush and rinsed with $10 \mathrm{~mL}$ of saline. About $20 \mathrm{~s}$ after injection, the green light was turned on to spot the margin of parenchymal resection. The margins were marked with the bipolar grasper. The grasper was previously placed in the right-hand port. Then, the specimen was placed in an Endobag.

The complementary section of the bronchus was done with curved scissors through the fissure exposure and sent for frozen section analysis.

For better control of the bronchial section, the third arm was anteriorly tracking the basal pyramid trunk with a silicone loop (10-cm cut blue loop).

As for ML anastomosis, the posterior approach was preferred as it ensured the natural encounter of the two borders and moved the artery away during suturing. The same steps of suture were performed as RUL anastomosis.

\section{Data analyses}

Quantitative data are presented as the number of observed values, mean \pm standard deviation, median, and range (min-max), while qualitative data are presented as the number of observed values. Complication severity was evaluated with Clavien Dindo classification ${ }^{[5]}$.

\section{RESULTS}

During this period (February 2014 to August 2019) in our institution, 582 patients underwent robotic thoracic procedures. In this cohort, 486 anatomical lung resections were performed, which involved 351 lobectomies or bilobectomies and 135 segmentectomies. Among these patients, 10 received a bronchial sleeve, i.e., $2 \%$ of the anatomical lung resections. The first bronchial sleeve was done on Patient 219. The main characteristics of the patients and procedures are reported in Table 1 in chronological order. The first 5 procedures were performed with the Da Vinci $\mathrm{Si}^{\mathrm{TM}}$ system and the last five with the Da Vinci $\mathrm{Xi}^{\mathrm{TM}}$ system.

All patient had accurate pathology diagnosis of the lesion preoperatively. All surgeries occurred on the right lung. None of the patients involved pathological nodes. All resection margins were Ro. The procedure details and outcomes are reported in Table 2 in the same order as Table 1.

No major events occurred preoperatively. Surgery duration was from 121 to $243 \mathrm{~min}$. No blood transfusion was required during hospital stay. No bronchial fistula occurred.

\section{DISCUSSION}

Bronchial sleeve procedures are complex and rare surgeries. The benefit of the enhanced vision and hand tool of the robotic system is significant for these surgeries. The principle of telemanipulation surgery is 
Table 1. Chronological description of the characteristics of patients and procedures

\begin{tabular}{|c|c|c|c|c|c|c|c|c|c|}
\hline Patient & Procedure & Sex & Age (years) & ASA & BMI & FEV (\%) & Tumor size (mm) & Number of nodes & Pathology \\
\hline 1 & $\mathrm{RUL}+\mathrm{ML}$ & $M$ & 36 & 2 & 27 & 99 & 25 & 20 & Carcinoid \\
\hline 2 & $M L$ & M & 62 & 3 & 27 & MD & 30 & 15 & $\mathrm{SCC}$ \\
\hline 3 & $M L+R I L$ & M & 17 & 1 & 22 & 85 & 12 & 6 & Carcinoid \\
\hline 4 & $\mathrm{ML}$ & $M$ & 52 & 3 & 21 & 107 & 30 & 21 & ADK \\
\hline 5 & RUL & $\mathrm{F}$ & 65 & 2 & 18 & 96 & 15 & 21 & SCC \\
\hline 6 & $S 6 R$ & M & 70 & 2 & 22 & 64 & 85 & 14 & SCC \\
\hline 7 & RIL & M & 60 & 3 & 25 & 75 & 20 & 25 & $\mathrm{SCC}$ \\
\hline 8 & RUL & M & 55 & 2 & 25 & 107 & 25 & 19 & Carcinoid \\
\hline 9 & $M L+R I L$ & M & 77 & 3 & 19 & 96 & 26 & 30 & SCC \\
\hline \multirow[t]{2}{*}{10} & RUL & M & 42 & 2 & 21 & 101 & 12 & 9 & Carcinoid \\
\hline & Median [Min; Max] & & $58[17 ; 77]$ & $2[1 ; 3]$ & $22[18 ; 27]$ & $96[64 ; 10]$ & $25[12 ; 85]$ & $19.5[6 ; 30]$ & \\
\hline
\end{tabular}

ML: median lobectomy; RUL: right upper lobectomy; RIL: right inferior lobectomy; S6R: segment 6 right lung; MD, missing data; SCC: squamous cell carcinoma; ADK: adenocarcinoma

Table 2. Details of patients' procedures, outcomes, and complications

\begin{tabular}{|c|c|c|c|c|c|c|c|c|c|}
\hline Patient & $\begin{array}{l}\text { Sleeve } \\
\text { procedure }\end{array}$ & N staplers & $\begin{array}{l}\text { Surgery } \\
\text { duration (min) }\end{array}$ & $\begin{array}{l}\text { Blood loss } \\
(\mathrm{mL})\end{array}$ & $\begin{array}{l}\text { Chest tube } \\
\text { (d) }\end{array}$ & $\operatorname{LOS}(d)$ & $\begin{array}{l}\begin{array}{l}\text { Complication } \\
\text { (yes 1, no 0) }\end{array} \\
\end{array}$ & Clavien dindo & Complication type \\
\hline 1 & $\mathrm{RUL}+\mathrm{ML}$ & 1 & 141 & 5 & 2 & 4 & 0 & 0 & \\
\hline 2 & $M L$ & 6 & 243 & 150 & 5 & 7 & 0 & 0 & \\
\hline 3 & $M L+R I L$ & 4 & 121 & 5 & 5 & 7 & 1 & 2 & Chylothorax \\
\hline 4 & $M L$ & 8 & 227 & 50 & 10 & 12 & 1 & 2 & Air leak $>5$ days \\
\hline 5 & RUL & 5 & 141 & 5 & 3 & 12 & 1 & 2 & Pneumothorax \\
\hline 6 & $S 6 R$ & 8 & 156 & 100 & 6 & 9 & 1 & 2 & Bronchitis \\
\hline 7 & RIL & 5 & 125 & 5 & 4 & 19 & 1 & 5 & Gastric hemorrhage \\
\hline 8 & RUL & 4 & 125 & 50 & 2 & 4 & 0 & 0 & \\
\hline 9 & $M L+R I L$ & 6 & 189 & 50 & 7 & 20 & 1 & 2 & Air leak $>5$ days \\
\hline \multirow[t]{2}{*}{10} & RUL & 3 & 176 & 150 & 4 & 7 & 1 & 2 & Atelectasis \\
\hline & Mean $( \pm$ SD) & $5( \pm 2.2)$ & $164( \pm 43)$ & $57( \pm 57)$ & $4.8( \pm 2.4)$ & $10( \pm 5.7)$ & & & \\
\hline
\end{tabular}

ML: median lobectomy; RUL: right upper lobectomy; RIL: right inferior lobectomy; S6R: segment 6 right lung; LOS: length of stay; SD: standard deviation

to allow open surgery procedures in a closed chest. This means that the procedure flow is the same as the open surgery gold standard.

In our experience, we have had no conversions. We found a longer LOS in this group rather than standard procedure or those described in ${ }^{[6]}$. First, patients requiring this procedure might have comorbidities and thus are at risk of complications, thereby requiring more hospital care. Second, the postoperative risk concerns mainly the scaring process on the bronchus, which requires a closer check of the patients and thus a longer length of stay. The aim of this surgery is not the quickest outcome but a good outcome that spares the lung. In our series, 1 patient died during hospital stay of massive gastric hemorrhage. After analyzing this case during a dedicated mortality meeting, this dramatic outcome was not found to be related to the surgical approach. The patients' comorbidities and the stress of such disease are real. This highlights the severity of the underlying pathologies and risks. We do not understate the harshness of disease and surgical risks due to miniaturization of thoracic penetration, especially for complex procedures.

Our 1st sleeve procedure was achieved for Patient 219, i.e., after significant experience with the machine. This might have given the surgeon time to be technically confident and therefore appropriate for the patient case. The learning curve for complex procedures depends on the surgeon's self-appreciation and cannot be estimated as in standard procedures at around 30 cases $^{[7]}$. For complex procedures, the surgeon's mastery of the tool is the 1st step and remains based on their honest capacity assessment. The other restricting element is patient selection. As shown in our experience, these are rare indications (2\%). We are aware of the patient 
benefit in terms of disease-free survival and do not push for technical achievement ${ }^{[8]}$. In our series of ten patients, 4 had carcinoid tumor, 5 had squamous cell carcinoma (SCC), and only 1 adenocarcinoma; none had node involvement. It makes sense, as SCC and carcinoid tumors are more proximal, endobronchial diseases than other tumors and might be more subject to such procedures.

The selection of patients is mandatory and we summarize some criteria below regarding patient status and tumor standard:

- Either poor lung function or patient's comorbidities to avoid pneumonectomy outcome ${ }^{[9]}$.

- Ro achievement.

- Low degree of aggressiveness of disease (carcinoid tumors and No disease).

In our experience, the tumor size is not a major limit as we have removed tumors with size up to $85 \mathrm{~mm}$. The distance between the tumor and the vascular structure is more limiting than the size itself. We are still waiting for appropriate tools to clamp either the pulmonary artery or the pulmonary vein to allow safe vascular sleeve resection. That might explain why we have only performed right-side bronchial sleeve, as most left-side bronchial sleeve cases require a proximal vascular control.

In our experience, it is a bloodless surgery [mean blood loss: $57 \mathrm{~mL}( \pm 57)$ ]. Hemostasis during the procedure is cautiously realized as the dryness of the operating field is mandatory to assess good vision (red color decreases brightness and contrast).

The patients were placed in lateral lying position without bending the table to avoid any limitation of the venous flow of the lower body, which combined with the capnothorax might trouble the cardiac input. The partial W-shaped port position ensures a non-conflicting position of the arms and instruments either outside or inside. It can be applied for any anatomical lung resection and is the same for the left side (mirror effect). The principle is to have the camera above the hands, similar to how the head is above the shoulders, and to have an assistant on the side coming perpendicularly. This setting avoids conflict and allows a complete control of the chest target zones. The $30^{\circ}$ vision is also important for providing an overview of the target and to avoid blind spots while twisting the camera. At the beginning of the procedure, the chest wall is viewed through $30^{\circ}$-up vision and the procedure is achieved through $30^{\circ}$-down vision. The third-hand position is also meant to avoid conflict with the left hand, inside or outside, as it enters $90^{\circ}$ to the chest, higher and closer to the spine.

For ML bronchial anastomosis, the exposure change (from fissure view for resection of the bronchus to posterior view behind the pulmonary vein) is of great interest for presenting the 2 bronchial borders and vanishing pulmonary artery away from the suturing zone. The versatility of exposure during robotic surgery must be exploited.

The use of barbed wire secures the tension adaptation of the running suture, but it is not mandatory. Previously published small series of patients have described the use of braided waxed sutures such as Vicryl running sutures or separate sutures and the use of monofilament such as polydioxanone ${ }^{[6,10-14]}$. These papers show similar outcomes to our series but do not describe our fully-closed-chest four-arm robotic technique. The use of absorbable monofilament might be tricky as its elasticity might be difficult to handle without haptic feedback. The choice of the needle is also important, as it must be small (17 mm) and semicircular to be scaled and fit to the instruments and bronchial structures. To limit the risk of wire rupture while knotting due to excess of "manual" tension, we suggest the use of the strongest V-Loc ${ }^{\mathrm{TM}}$, i.e., 180. Even then, the surgeon must be aware of this risk and be as delicate as possible while applying distraction force on the wire. The barbed suture does not require more than four knots to be stable, which is fewer than monofilament wires. The lack of haptic feedback is balanced by enhanced vision in most situations. 
The size of the wire must not be too thick to prevent the cut of the borders. Because of magnification, the surgeon might tend to take smaller border margins and quicker steps to progress than in open surgery during the running suture. It might then be useful to pay attention to the size of the instruments to keep an accurate scale evaluation of measure.

The surgeons' hard skills are conserved, indeed enhanced, by the telemanipulator and favor the precise and tremorless moves of the instruments for either forehand or backhand sutures (bimanual surgery). This technical improvement ensures manual knot and stitch of distal arteries, which are a key point of these advanced procedures. First, it is the basis of the surgeon's hard skills development and retention. Second, it helps for control and exposure of vascular structures when required. A stapleless artery is safer to manipulate and the presence of a nude arterial stump to hold can be useful to mobilize/expose the artery.

During our experience, we switched from the Da Vinci ${ }^{\mathrm{TM}}{ }^{\mathrm{M}}$ system to the $\mathrm{X} i{ }^{\mathrm{TM}}$ system. The benefit of this upgrade is valuable for the surgeon and the operating room setting. With the $\mathrm{Si}^{\mathrm{TM}}$ system, we were using a $12-\mathrm{mm}$ camera to ensure the best vision; with the $\mathrm{X} i{ }^{\mathrm{TM}}$ system, the full high definition vision is provided through an $8-\mathrm{mm}$ camera, and smaller is better for the patient's postoperative pain. The $\mathrm{Si}^{\mathrm{TM}}$ system needed to be placed on the axis of the scapula line, which limited the head access for the anesthesiologist during the procedure and prolonged the procedure setting. The $\mathrm{X}{ }^{\mathrm{TM}}$ system has smaller and wider motion zone moves than the $\mathrm{Si}^{\mathrm{TM}}$ system, as well as an autofocus camera and surgeon console control of the upsidedown twist of camera, which might save time and comfort during the procedure. This might explain the decreasing trend of procedure lengths between the first half and second half of our series.

In our experience, four-arm robotic bronchial sleeve lung resection and node harvest is safe and feasible. Patient selection and surgeon's robotic expertise are mandatory to perform such rare procedures. Dedicated vascular clamping devices are awaited to enlarge indications to left side and vascular sleeve resections.

\section{DECLARATIONS}

\section{Authors' contributions}

The author contributed solely to the article.

\section{Availability of data and materials}

Not applicable.

\section{Financial support and sponsorship}

None.

\section{Conflicts of interest}

Author is an official proctor for Intuitive Surgical.

\section{Ethical approval and consent to participate}

Not applicable.

\section{Consent for publication}

Not applicable.

\section{Copyright}

(c) The Author(s) 2019. 


\section{REFERENCES}

1. Li C, Han Y, Han D, Chen X, Chen K, et al. Robotic approach to combined anatomic pulmonary subsegmentectomy: technical aspects and early results. Ann Thorac Surg 2019;107:1480-6.

2. Shanahan B, O’Sullivan KE, Redmond KC. Robotic sleeve lobectomy-recent advances. J Thorac Dis 2019;11:1074-5.

3. Durand M, Dabboura E, Lamonerie L, Herkert A, Zarka V, et al. Four-arm robotic lung resection versus muscle-sparing minithoracotomy: retrospective experience. J Thorac Dis 2019;11:1433-42.

4. Durand M. Four-arm robotic sleeve right upper lobectomy. Ann Cardiothorac Surg 2019;8:286-7.

5. Dindo D, Demartines N, Clavien PA. Classification of surgical complications: a new proposal with evaluation in a cohort of 6336 patients and results of a survey. Ann Surg 2004;240:205-13.

6. Jiao W, Zhao Y, Qiu T, Xuan Y, Sun X, et al. Robotic bronchial sleeve lobectomy for central lung tumors: technique and outcome. Ann Thorac Surg 2019;108:211-8.

7. Song G, Sun X, Miao S, Li S, Zhao Y, et al. Learning curve for robot-assisted lobectomy of lung cancer. J Thorac Dis 2019;11:2431-7.

8. Reddy RM. Robotic sleeve lobectomy-technically possible but is it being overused? J Thorac Dis 2019;11:S1211-2.

9. Pages PB, Mordant P, Renaud S, Brouchet L, Thomas PA, et al. Sleeve lobectomy may provide better outcomes than pneumonectomy for non-small cell lung cancer. A decade in a nationwide study. J Thorac Cardiovasc Surg 2017;153:184-95. e3.

10. Cerfolio RJ. Robotic sleeve lobectomy: technical details and early results. J Thorac Dis 2016;8:S223-6.

11. Lin MW, Kuo SW, Yang SM, Lee JM. Robotic-assisted thoracoscopic sleeve lobectomy for locally advanced lung cancer. J Thorac Dis 2016;8:1747-52.

12. Pan X, Gu C, Wang R, Zhao H, Shi J, et al. Initial experience of robotic sleeve resection for lung cancer patients. Ann Thorac Surg 2016;102:1892-7.

13. Gu C, Pan X, Chen Y, Yang J, Zhao H, et al. Short-term and mid-term survival in bronchial sleeve resection by robotic system versus thoracotomy for centrally located lung cancer. Eur J Cardiothorac Surg 2018;53:648-55.

14. Li C, Zhou B, Han Y, Jin R, Xiang J, et al. Robotic sleeve resection for pulmonary disease. World J Surg Oncol 2018;16:74. 